\title{
Lithography process simulation studies using coarse-grained polymer models
}

\author{
Hiroshi Morita
}

Lithography processes were modeled on the basis of coarse-grained polymer simulation techniques using OCTA, which is the simulation software for soft materials, and the simulations of these processes were performed to clarify the mechanism of the polymer chain dynamics in lithography processes. In the case of the top-down process, the development and rinse processes were modeled using a dissipative particle dynamics method. From our series of simulations, the line pattern constituted by resist polymers can be simulated as a result. In the rinse process, the reduction of line edge roughness (LER) can be found, and the importance of the rinse process to modify the LER was identified. The bottom-up process, which is called the directed selfassembly (DSA) process, can also be simulated. From our DSA process simulations, the polymer chain dynamics in the defect annihilation process can be analyzed.

Polymer Journal (2016) 48, 45-50; doi:10.1038/pj.2015.78; published online 30 September 2015

\section{INTRODUCTION}

For integrated semiconductors, the patterning width in the semiconductor circuit is becoming increasingly smaller, and recently the width has become smaller than $20 \mathrm{~nm} .^{1}$ The smallest patterning width used in the industrial process is now $22 \mathrm{~nm}$, and that on the laboratory level is $<10 \mathrm{~nm}$ when using multiple patterning, directed self-assembly (DSA) or extreme ultraviolet (EUV) patterning methods. As the width decreases, it becomes closer to the size of resist polymer, and it is difficult to develop suitable photoresist polymers for these processes.

In addition, the simulations of polymeric materials of $\sim 20 \mathrm{~nm}$, which is in the range of the 'meso-scale,' can be performed using the OCTA system, which was developed by Masao Doi and coworkers. ${ }^{2}$ Since 2002, when the first version of OCTA was released, simulation studies for polymeric materials have been frequently reported, and OCTA has also been used by many researchers. Currently, the total number of registered OCTA users is $\sim 1700$. Using the OCTA system, it became much easier to simulate polymeric materials.

Because the pattern width size of the semiconductor is now in the range of meso-scale simulation, it is possible for us to perform simulation studies of the lithography process, and in 2008 our research of the lithography process simulation was started. ${ }^{3-10}$ First, the development and the rinse processes in the top-down process were simulated, ${ }^{3-6}$ and recently the bottom-up processes, ${ }^{7-10}$ such as the DSA process, were simulated using the OCTA system. In this review paper, the lithography process simulations are introduced, and the applicability of our simulation models and methods is discussed. First, we explain the application to the top-down process, such as the development and the rinse processes. Next, as a bottom-up process, the application to the DSA process is explained. All of these studies have been performed using the original OCTA system.

\section{METHODS}

The simulations indicated in this paper have been performed using the dissipative particle dynamics (DPD) method proposed by Groot and Warren. ${ }^{11}$ The polymer chains are coarse-grained, DPD particle chains connected by springs, and the model of the system for the development, the rinse and the DSA processes were constructed by these coarse-grained polymers. There are several features of the DPD method. Because the weak interaction is treated in the non-bonding interaction potential in the DPD method, the polymer chains in the DPD method do not entangle and are modeled as phantom chains. Therefore, using the DPD method, we can perform a long time simulation over the entanglement time and integrate the simulationrelated phase transition. All simulations were performed using the COGNAC/OCTA simulator, ${ }^{12}$ which is a general coarse-grained molecular dynamics simulator. In the latter subsections, the details of our simulation method are described.

\section{DPD method}

In this subsection, an overview of the DPD method is provided. $\mathbf{r}_{i}, v_{i}$ and $m_{i}$ are the position, velocity, and mass of the $i$-th particle, respectively, and each particle obeys the equation of motion, ${ }^{11}$

$$
\begin{aligned}
& \frac{\mathrm{d} \mathbf{r}_{i}}{\mathrm{~d} t}=\mathbf{v}_{i} \\
& m_{i} \frac{\mathrm{d} \mathbf{v}_{i}}{\mathrm{~d} t}=\mathbf{f}_{i}
\end{aligned}
$$


where $t$ is time, and $\mathbf{f}_{i}$ is the force acting on the $i$-th particle. $\mathbf{f}_{i}$ is calculated by the summation of three terms,

$$
\mathbf{f}_{i}=\sum_{j \neq i}\left(\mathbf{F}_{i j}^{\mathrm{C}}+\mathbf{F}_{i j}^{\mathrm{D}}+\mathbf{F}_{i j}^{\mathrm{R}}\right)
$$

where $\mathbf{F}_{i j}^{\mathrm{C}}, \mathbf{F}_{i j}^{\mathrm{D}}$ and $\mathbf{F}_{i j}^{\mathrm{R}}$ are the conservative force, dissipative or drag force and random force, respectively. In $\mathbf{F}_{i j}^{C}$, the interactions due to bonding and non-bonding are included,

$$
\begin{aligned}
& \mathbf{F}_{i j}^{C}=\mathbf{f}^{\text {bond }}\left(\mathbf{r}_{i j}\right)+\mathbf{f}^{\text {non-bond }}\left(\mathbf{r}_{i j}\right) \\
& \mathbf{f}^{\text {bond }}\left(\mathbf{r}_{i j}\right)=\frac{1}{2} \mathbf{C r}_{i j}^{2} \\
& \mathbf{f}^{\text {non-bond }}\left(\mathbf{r}_{i j}\right)=\left\{\begin{array}{cc}
\frac{1}{2} a_{i j}\left(1-\frac{\mathbf{r}_{i j}}{\mathbf{r}_{\mathrm{c}}}\right)^{2} & \left(\mathbf{r}_{i j}<\mathbf{r}_{\mathrm{c}}\right) \\
0 & \left(\mathbf{r}_{i j} \geq \mathbf{r}_{\mathrm{c}}\right)
\end{array}\right.
\end{aligned}
$$

where $r_{\mathrm{c}}$ is the cut-off distance, $a_{i j}$ is the maximum repulsion between particle $i$ and $j$, and $\mathbf{r}_{i j}=\mathbf{r}_{i}-\mathbf{r}_{j}$. The dissipative or $\operatorname{drag}\left(\mathbf{F}_{i j}^{\mathrm{D}}\right)$ and random $\left(\mathbf{F}_{i j}^{R}\right)$ forces are given by

$$
\begin{aligned}
& \mathbf{F}_{i j}^{\mathrm{D}}=-\gamma \omega^{\mathrm{D}}\left(\mathbf{r}_{i j}\right)\left(\mathbf{v}_{i j} \cdot \frac{\mathbf{r}_{i j}}{\mathbf{r}_{i j}}\right) \frac{\mathbf{r}_{i j}}{\mathbf{r}_{i j}} \\
& \mathbf{F}_{i j}^{\mathrm{R}}=\sigma \omega^{\mathrm{R}}\left(\mathbf{r}_{i j}\right) \theta_{i j} \frac{\mathbf{r}_{i j}}{\mathbf{r}_{i j}}
\end{aligned}
$$

where $\theta_{i j}$ is a randomly fluctuating variable with Gaussian statistics, and $w_{\mathrm{D}}$ and $w_{\mathrm{R}}$ are $r$-dependent weight functions, which are given by

$$
\omega^{\mathrm{D}}\left(\mathbf{r}_{i j}\right)=\left[\omega^{\mathrm{R}}\left(\mathbf{r}_{i j}\right)\right]^{2}=\left\{\begin{array}{cc}
\left(1-\frac{\mathbf{r}_{i j}}{\mathbf{r}_{\mathrm{c}}}\right)^{2} & \left(\mathbf{r}_{i j}<\mathbf{r}_{\mathrm{c}}\right) \\
0 & \left(\mathbf{r}_{i j} \geqslant \mathbf{r}_{\mathrm{c}}\right)
\end{array}\right.
$$

In our simulations, the other parameters used include $\gamma=4.5$, $m=k_{\mathrm{B}} T=r_{\mathrm{c}}=1, a_{i i}=25$ and

$$
\sigma^{2}=2 \gamma k_{\mathrm{B}} T
$$

\section{Model of resist polymer}

Figure 1 shows the coarse-grained polymer chain model in our simulation based on the DPD method. The polymer chain is represented as DPD particles connected by springs, and a linear chain is formed. Each particle contains several monomers, and its size is $\sim 1-2 \mathrm{~nm}$ on the realistic length scale. These particles interact with each other, and the affinity is set by $a_{i j}$, which is the non-bonding interaction parameter in equation (6). If $a_{i j}$ is larger, the interaction between $i$-th and $j$-th types of particles becomes more repulsive. If $a_{i j}$ is smaller than $a_{i i}$ or $a_{i j}$, the particles attractively interact. In the case of the development process simulation, by setting smaller $a_{\mathrm{ps}}$, where $\mathrm{p}$ and $s$ are the polymer and solvent, respectively, the polymers dissolve into the solvent phase. In the case of the DSA simulation, the polymer contains two types of blocks, and a larger $a_{i j}$ between $i$ and $j$ species is set. As a consequence, micro-phase-separated structures are formed.

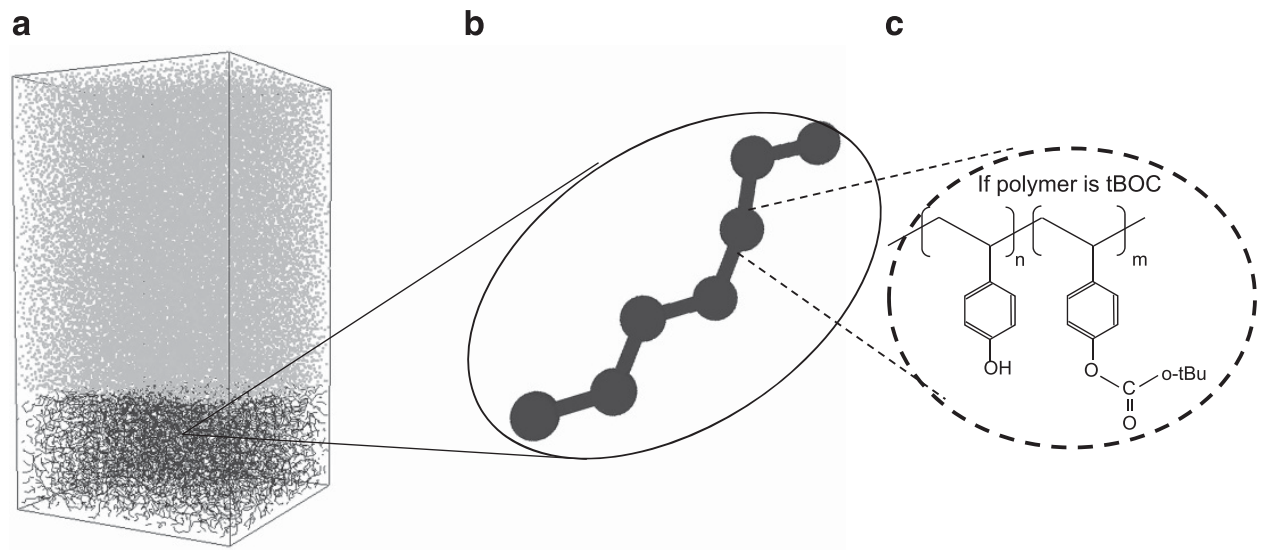

Figure 1 Coarse-grained polymer chain model used in the lithography process simulations. ( $\mathbf{a}$ and $\mathbf{b}$ ) The total structure of the polymer film and the coarsegrained polymer chain. (c) An example of the poly(p-t-butyloxycarbonyloxystyrene) polymer chain corresponding to the coarse-grained polymer chain.

a

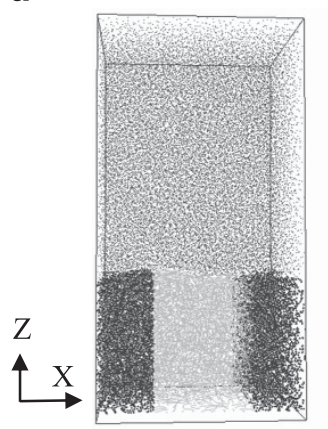

b

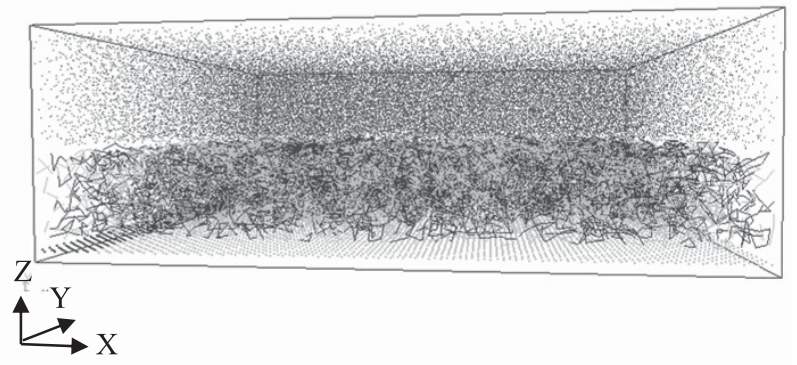

Figure 2 Initial structures of (a) top-down and (b) bottom-up process simulations. 
Simulation system for the development and the rinse processes Figure 2a shows the initial structure of the development process simulation. In the simulation box, the air and the polymer particles are filled, and a resist film on the substrate is spread in the $x-y$ direction over the lower region of the system. The resist polymer surface is represented by the interface between the air and the polymer layers. In the development and the rinse processes, the air particles are replaced with solvent particles, and the dissolution process is performed. In this paper, both air and solvent particles are denoted as 'S.' Simulations are conducted using a box of $20.64 r_{\mathrm{c}} \times 20.8539 r_{\mathrm{c}} \times 37.1726 r_{\mathrm{c}}$, where $r_{\mathrm{c}}$ is the cut-off distance of the interaction parameter in equation (6). The periodic boundary condition is applied for the $x-y$ direction. The boundary condition in $z=0$ (the condition for the substrate) is the wall condition, in which the Lennard-Jones potential is applied. At $z=z_{\max }$, the reflective boundary condition is applied.

The initial structure shown in Figure 2a is obtained by the densitybiased Monte Carlo method ${ }^{13}$ (DBMC). Details of the method are provided in ref. 13. In the DBMC method, the polymer chains are generated by the probability of each segment obtained by selfconsistent field simulation, ${ }^{14-16}$ and the local distribution of polymer particles near the surface or the interface can be set. For example, the segregation of the end particles of the polymer can be achieved using the DBMC method. In the simulation box, there are 1600 chains with chain length $N=10$ and 32000 air particles (S).

\section{Simulation system for the DSA process}

Figure $2 \mathrm{~b}$ shows a snapshot of the initial structure of the DSA simulation. This simulation uses an A-B diblock copolymer, in which 10 particles of segment $\mathrm{A}$ and 10 particles of segment B are connected. In the top layer, the air particles (S) are distributed in the same way as Figure 2a. In the middle layer, randomly distributed block copolymers are placed using the DBMC method. In the bottom layer, two types of particles are allied on the lower boundary, and a flat chemoepitaxy layer is modeled. One particle, w1, is attractive to A species of the copolymer, and another particle, w2, interacts neutrally with both A and B species. In our simulation, 1680 particles of w1, 18480 particles of w2, 1208 chains of copolymer and 40320 particles of S are contained in the simulation system. The same boundary conditions indicated in section 'Model of resist polymer' are applied. The size of the $x, y$ and $z$ (height) directions are $40 r_{\mathrm{c}}, 42 r_{\mathrm{c}}$ and $14 r_{\mathrm{c}}$, respectively. The values of the interaction parameters are given below, $a_{\mathrm{AB}}=60$, $a_{\mathrm{AA}}=a_{\mathrm{BB}}=25, a_{\mathrm{SA}}=a_{\mathrm{SB}}=200, a_{\mathrm{Aw} 1}=10$ and $a_{\mathrm{Bw} 1}=a_{\mathrm{Aw} 2}=a_{\mathrm{Bw} 2}=25$.

\section{RESULTS OF THE DEVELOPMENT AND RINSE PROCESS SIMULATION}

\section{Parameter check of the interaction parameter}

The interaction parameter $a_{\mathrm{ps}}$ is the repulsive non-bonding interaction parameter between the polymer and the solvent particles, and the solubility of the polymer in the solvent is controlled using $a_{\mathrm{ps}}$. As $a_{\mathrm{ps}}$ increases, the repulsive force increases, and the polymers do not dissolve. However, when $a_{\mathrm{ps}}$ is smaller than $a_{\mathrm{pp}}$ (interaction between polymers), the polymers can dissolve in the solvent. The solubility of the polymers can be checked by the parameter study of the interaction parameter $a_{\mathrm{ps}}$ in Figure 3. Figure 3 shows the snapshots of our simulations with parameter $a_{\mathrm{ps}}$ at time $=10000 \tau$. In the case of smaller $a_{\mathrm{ps}}$, the polymers can dissolve, whereas with larger $a_{\mathrm{ps}}$, the polymer cannot diffuse into the solvent phase. The transition between the soluble and the insoluble cases occurs suddenly between an $a_{\mathrm{ps}}$ of 20 and 25, and this transition is a first-order transition. In the following simulations, values of $a_{\mathrm{ps}}$ for soluble and insoluble polymer layers are set based on the result shown in Figure 3.

\section{Development process simulation}

Next, the simulations of the development process were performed. As discussed in the previous section, the solubility of the polymers can be controlled by the interaction parameter $a_{\mathrm{ps}}$. In our simulation, line pattern simulation was used. In the real industrial process, before the development step, photo-exposure is performed to create contrast between soluble and insoluble lines, and the width of the interface is determined by the length of the diffusion of PAG. The width is strongly related to the roughness of the line. In this simulation, the interfacial width is set as 0 , and the soluble and the insoluble particles are decided by the positions along the $x$ direction shown in Figure 2a. If the $\mathrm{x}$ position of the DPD particle is within half of the middle region in the simulation box, the particle is set as a soluble particle, and $a_{\mathrm{ps}}$ is inputted as 5. Other particles are set as insoluble particles, and $a_{\mathrm{ps}}$ is inputted as 45. Figure 4a shows the initial structure of the line pattern simulation. The simulation size used in our model is an $\sim 10-\mathrm{nm}$ film thickness and a $20-\mathrm{nm}$ periodic length line pattern. The middle line is the soluble line in the development simulation.

Figure 4 shows the results of the dissolution process simulation. As times passes, only the polymers in the soluble lines diffuse into the solvent phase, and the insoluble lines remain. In the middle soluble region, swelling occurs first, and then diffusion of the polymer proceeds. These structures can also be observed experimentally by fast atomic force microscopy (AFM) observation. Itani et al. ${ }^{17}$ performed fast AFM observation of the dissolution front of the line pattern system. From

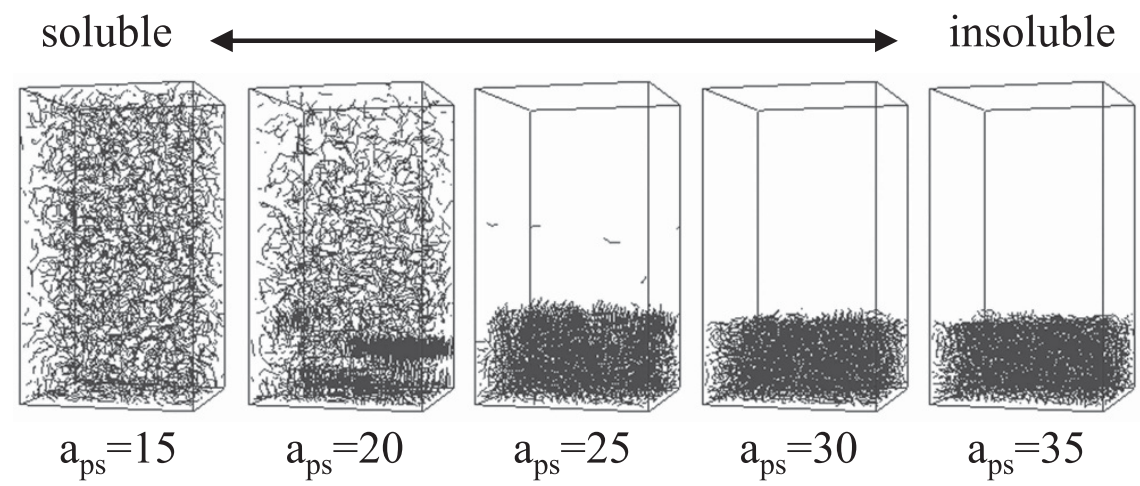

Figure 3 Snapshots of the results of the dissolution process simulations along with the interaction parameter $a_{\mathrm{ps}}$. 
a

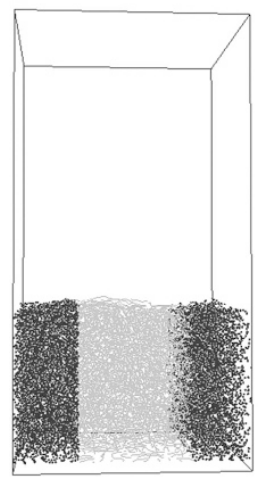

b

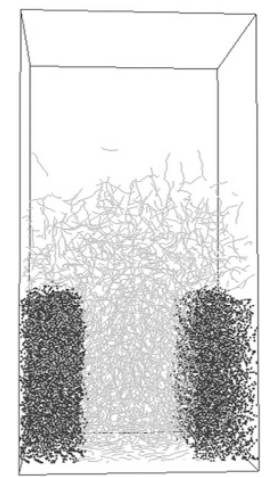

c

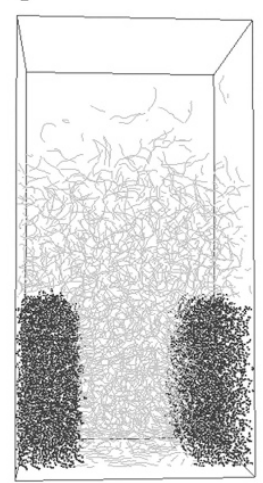

d

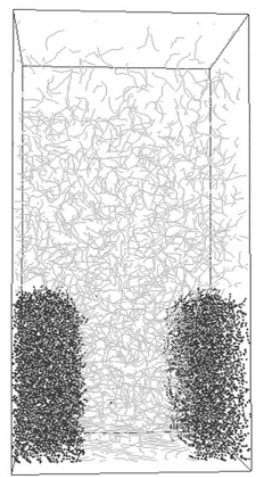

Figure 4 Results of the development process simulation in a line pattern at $t=0$ (a), 500 (b), 1000 (c) and 2500 (d).

a

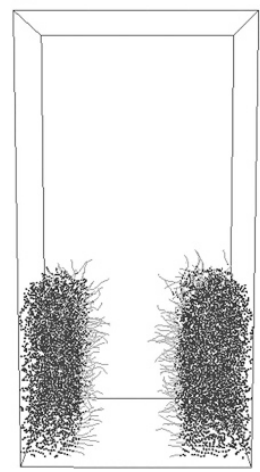

b

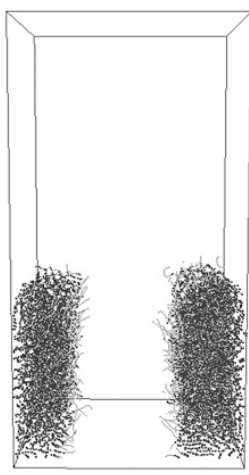

C

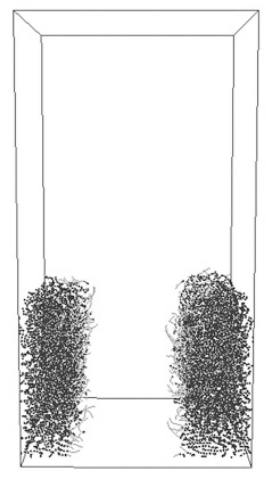

d

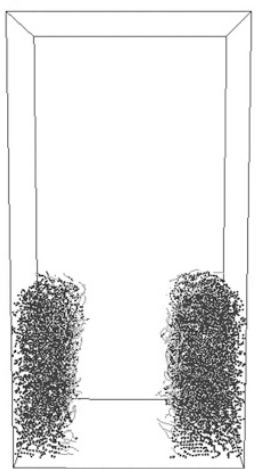

Figure 5 Results of the rinse process simulation in a line pattern at $t=0$ (a), 10 (b), 20 (c) and 50 (d).

their results, a similar structure in the swelling and the diffusion steps can be observed, and the reliability of our simulation can be confirmed.

In these simulations, the line edge roughness at each time step can also be analyzed. In the initial conditions, a flat interface is assumed, and the roughness is almost zero. As the dissolution of polymer proceeds, the roughness increases. The line edge is constituted by polymer chains attached to the residual line pattern, and the fluctuation of these polymers is considered as one of the reasons for the increasing reduction of line edge roughness (LER) on the meso-scale.

\section{Rinse process simulation}

Next, the rinse process simulation is conducted. After the development process simulation, there are many residual resist polymers in the system. The residual resist polymer chain is extracted and replaced by same number of solvent particles. Figure $4 \mathrm{~d}$ is the snapshot of the final steps of the development process simulation. Free polymer chains that do not attach to the line pattern are extracted from the simulation box. After these treatments, the structure shown in Figure 5a is obtained, which is the initial structure of the rinse process simulation.

Figure 5 shows the results of the rinse process simulation. The rinse liquid or air is distinguished as a poor solvent for the resist polymer. In the rinse process simulation, the repulsive interaction between the polymer and solvent is imposed as $a_{\mathrm{ps}}=45$. Although stretched-out chains near the line edge can be observed in the last step of the dissolution process, these chains are instantly shrunk in the rinse process.

The LER is improved in the rinse process. Once the polymer chains at the line edge contact poor solvent, the polymer chains shrink due to the decreased interface between the polymer and poor solvent. As a result, the interface becomes flattened. This result indicates that LER can be modified in the rinse process.

\section{Correspondence to real experiment}

One of the disadvantages of our simulation using the DPD model is the uncertainty of the units (length, time and so on) due to the coarsegraining. For example, $r_{\mathrm{c}}$ is the unit of length, and we know that $1 r_{\mathrm{c}}$ roughly corresponds to $1-2 \mathrm{~nm}$ on an empirical basis. However, the correspondences of the length or other units were not clearly fixed in our previous studies. To map our simulations to the real polymer system, we must study each correspondence of each unit for each polymer. The correspondence between real experiments and our simulations is important for us to make our simulations meaningful.

As an example of a mapping study between simulation and experiment, the dissolution rate is investigated. With changing interaction parameters, dissolution process simulations of the polymer can be achieved. As the interaction parameter between the polymer and the solvent becomes more attractive than the interaction between polymers, the polymer can dissolve into the solvent much faster. For the simulation study, while changing the interaction parameters, the dissolution rate, which is defined as the change of the total density of the polymer film, is estimated. The experiment involves dissolution experiments of acryl resist polymer film into butyl acetate or 2-heptanon, and the experimental results of the dissolution rate are obtained. In the experiments, the dissolution rate is estimated by the change of the film thickness over time in the dissolution experiment. The best fits to the experimental results are chosen within the 
simulation results while changing the interaction parameters, and the optimum sets for the systems of acryl resist polymer/butyl acetate and acryl resist polymer/2-heptanon are obtained.

Figure 6 shows the best-fitted results of the dissolution rate as a function of the resist polymer's molecular weight. The simulation result using the parameter set of $a_{\mathrm{sp}}=25.0$ and $a_{\mathrm{ss}}=23.0$ is suitable for the result of the dissolution of the butyl acetate system, and the result using the parameter set of $a_{\mathrm{sp}}=25.0$ and $a_{\mathrm{ss}}=25.0$ is optimal for that of the 2-heptanone system. Once we obtain the fitted parameters, we

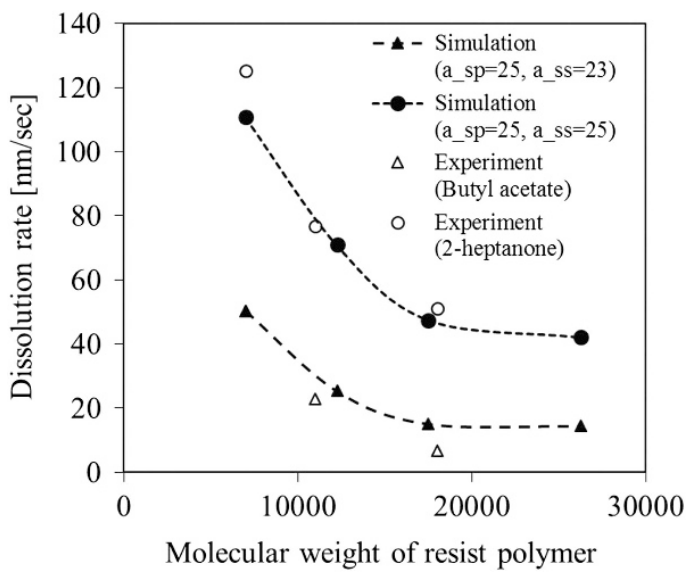

Figure 6 Comparison of the dissolution rate between experiments and simulations. can perform the corresponding simulation, and the virtual experiment using DPD simulation can be done. The LER using these parameters is discussed in Morita et al. ${ }^{6}$

\section{RESULTS OF THE DSA PROCESS SIMULATION}

\section{Dynamical process simulation}

OCTA is also applicable to the simulation study of another technique for next generation lithography, which is the DSA method. The DSA method is a block copolymer lithography technique, in which the micro-phase-separated structure of the film, such as the lamellar structure, is used as the line and space structure. Using the initial structure shown in Figure 2b, DSA process simulations can be performed. Non-bonding interactions between the particles of each block are represented by the parameter $a_{i j}$ in equation 6 , and these values were given in the section 'Simulation system for the development and the rinse processes'.

Figure 7 shows one result of the DPD simulation. In this figure, the top-down images are shown as time passes from left to right. Figure 7a shows the images constituted by polymer chains. Because it is difficult to understand the detail interfacial structures between the A and $\mathrm{B}$ domains in Figure $7 \mathrm{a}$, the interface, where the density of the A segment is 0.5 , is shown in Figures $7 \mathrm{~b}-\mathrm{f}$ and Figure 8. Because the wetting layer is in the direction along the $y$ axis (in this figure, the $y$ axis is the vertical direction), the lamellar is aligned with the $y$ axis, and an ordered lamellar structure is obtained at time $=1500$. In the series of figures, many defect annihilations are found, which are analyzed using the dynamics of the end particle in the next section.

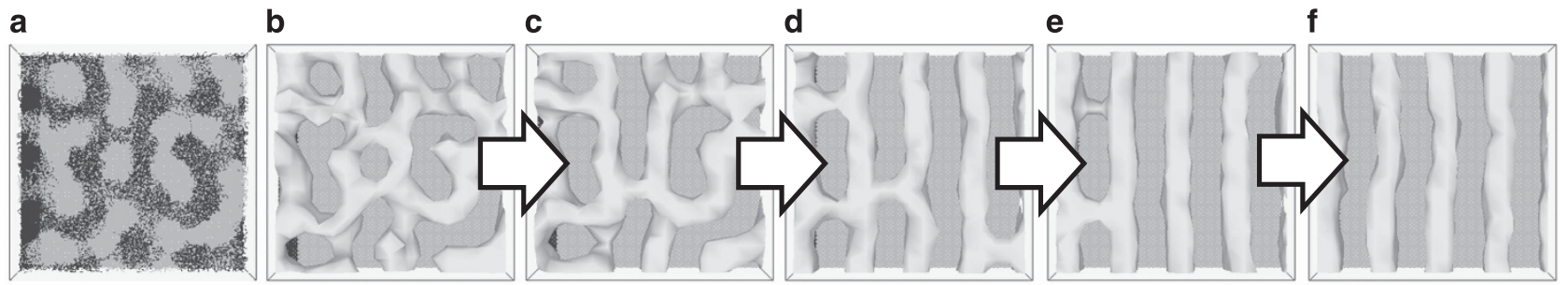

Figure 7 Initial structure in a polymer chain system (a) and the results of the DSA process simulation at $t=10$ (b), 200 (c), 500 (d), 1000 (e) and 2000 (f). DSA, directed self-assembly.

a
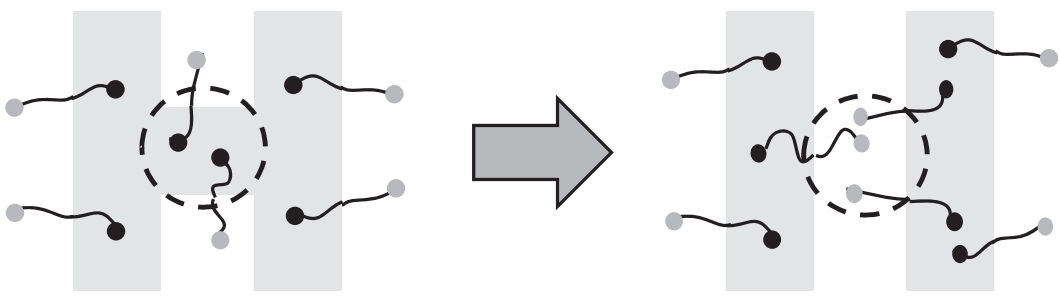

b

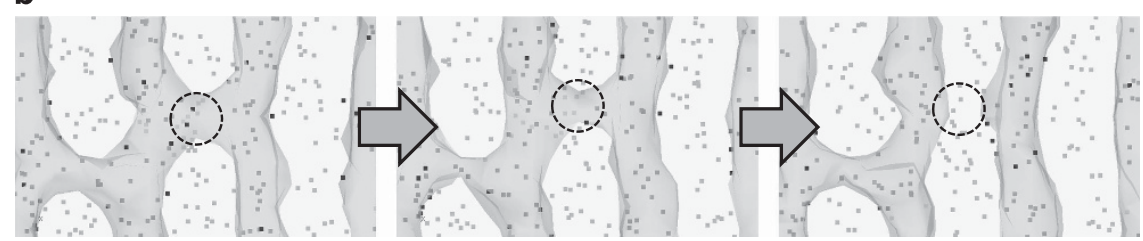

Figure 8 Defect annihilation process in a model structure (a) and the simulation result (b). (a) Cartoon of the polymer chain conformation in the defect and ordered structures, and (b) the dynamics of the defect annihilation steps in the DSA process simulation. DSA, directed self-assembly. 


\section{Analysis of polymer chain dynamics}

In the defect annihilation process, each polymer in the film moves and the conformations of some polymers at defect regions are changed. The ends of the polymer are the high entropy parts of the polymer chain and are the most mobile parts in the reputation dynamics of the polymer chain in the theories of polymer dynamics. ${ }^{18}$ The dynamics of ends act in advance of the other part of the polymer chain, and we focus on the dynamics of the end segments in the defect annihilation process. Figure $8 \mathrm{a}$ shows representations of the polymer chains in structures with and without defects. Junctions of A and B blocks are distributed near the interface locally, and both ends are distributed in the middle region of the domains. In the defect structure, the A domain is bridged between two lamellar domains, and the ends of the A block are distributed in the focused area indicated by the circle. In the final ordered structure, the ends of the B block are distributed in the same area. Therefore, in the defect annihilation process, in the focused area, the extraction of the ends of the A block occurs first, and the approach of the ends of the B block follows.

To verify our assumption of the dynamics of the ends, the positions of the ends in the defect annihilation process are analyzed. Figure $8 \mathrm{~b}$ shows the analysis of the ends in the defect annihilation process. The ends of the A and B domains are indicated by black and gray particles, respectively. In the left figure, black particles are found in the bridged region indicated by the circle; in the middle figure, almost all the black particles are extracted from this region; and in the right figure, the defects are annihilated and the black particles exit this region. Similar dynamics of the end particles are found in other defect annihilation processes. This result indicated that the dynamics of the ends of the polymer is very important in the defect annihilation process, and the observation of the ends provides helpful information about the defect annihilation process.

\section{CONCLUDING REMARKS}

In this review paper, simulation studies related to the lithography process are described. In the first topic, a top-down lithography process simulation is performed. Using the coarse-grained polymer model, the development and the rinse processes are simulated. In these simulations, the control of the conformation of the polymer chain at the line edge is important for decreasing the LER. In the rinse process, the swelling polymer of the line edge shrinks, and the modification of the LER can be simulated. Furthermore, the optimum parameter sets for the real system are obtained by mapping the dissolution rate. Using these parameters, we perform virtual experiments for the development and rinse processes of the lithography process.

In the second topic, a bottom-up process, the DSA process, is simulated using the DPD method. In the DPD simulation, we follow the dynamics of the polymer chains. In our studies, the ends of the block copolymer are focused on to analyze the defect annihilation process. In the defect annihilation process, the characteristic movement of the end particles in the defect region is determined.

The simulations in these studies were performed using the same simulator, OCTA/COGNAC, and these studies indicated that OCTA is applicable for these lithography process simulations. These applications are only a few examples, and in the future further application studies using OCTA will be published.

\section{CONFLICT OF INTEREST}

The author declares no conflict of interest

\section{ACKNOWLEDGEMENTS}

We would like to thank Intel Corporation, the New Energy and Industrial Technology Development Organization (NEDO) of Japan and EIDEC for the financial support of these studies. We would also like to thank the collaborator of these studies for many helpful comments and discussions.

1 ITRS working group. International Technology Roadmap for Semiconductors. http://www.itrs.net/. (2013).

2 Doi, M., OCTA http://octa.jp/. (2002) Accessed 4 May 2015.

3 Morita, H. \& Doi, M. Meso-scale simulation of the polymer dynamics in the formation process of line-edge roughness. Proc. SPIE 7273, 727337 (2009).

4 Morita, H. \& Doi, M. Mesoscale simulation of line-edge structures based on polymer chains in development and rinse processes. J. Micro/Nanolith. MEMS MOEMS. 9, 041213 (2010).

5 Morita, H. Meso-scale simulation of the line-edge structure based on resist polymer molecules by negative-tone process. Proc. SPIE 7972, 79720W (2011).

6 Morita, H., Okabe, I., Agarwal, S. \& Singh, V. K. Mesoscale simulation of the line-edge structure during positive and negative tone resist development process. Proc. SPIE 8325, 83250J (2012).

7 Morita, H. Applicable simulation methods for directed self-assembly -advantages and disadvantages of these methods. J. Photopolymer Sci. Technol. 26, 801-807 (2013).

8 Morita, H. Development of fast DSA simulation method using OCTA system. Proc. SPIE 9049, 904920 (2014).

9 Morita, H. Lithography process simulations using OCTA -application to development and DSA. J. Photopolymer Sci. Technol. 27, 25-29 (2014).

10 Morita, H. \& Norizoe, Y. Analysis of the self-assembling and the defect annihilation processes in DSA using meso-scale simulation. Proc. SPIE 9425, 942510 (2015).

11 Groot, R. D. \& Warren, P. B. Dissipative particle dynamics: bridging the gap between atomistic and mesoscopic simulation. J. Chem. Phys. 107, 4423-4435 (1997).

12 Aoyagi, T., Sawa, F., Shoji, T., Fukunaga, H., Takimoto, J. \& Doi, M. A general-purpose coarse-grained molecular dynamics program. Computer Phys. Commun. 145, 267-279 (2002).

13 Aoyagi, T., Honda, T. \& Doi, M. Microstructural study of mechanical properties of the ABA triblock copolymer using self-consistent field and molecular dynamics. J. Chem. Phys. 117, 8153-8161 (2002).

14 Matsen, M. W. \& Schick, M. Stable and unstable phases of a diblock copolymer melt. Phys. Rev. Lett. 72, 2660-2663 (1994).

15 Helfand, E. \& Wasserman, Z. R. Block copolymer theory. 4. Narrow interphase approximation. Macromolecules 9, 879-888 (1976).

16 Fleer, G. J., Cohen Stuart, M. A., Scheutjens, J. M. H. M., Cosgrove, T. \& Vincent, B. Polymers at Interfaces (Chapman \& Hall, London, UK, 1993).

17 Itani, T. \& Santillan, J. Dissolution behavior of photoresists: an in-situ analysis. J. Photopolymer Sci. Technol. 23, 639-642 (2010).

18 Doi, M. \& Edwards, S. F. The Theory of Polymer Dynamics (Oxford Scientific Publications, Oxford, UK, 1986).

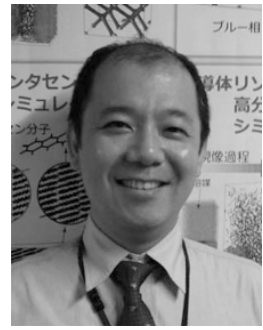

Dr Hiroshi Morita was born in Kobe, Japan in 1969. He received his Doctoral degree of Engineering in 1997 from Kyoto University. In the same year, he joined Japan synthetic rubber company (present JSR Company). From 1998 to 2000, he was on loan to JCII and joined Doi's project at Nagoya University to develop OCTA system. He was moved to The University of Tokyo as a researcher in 2002. From 2003, he was transferred to JST as a researcher and worked at Nagoya University and The University of Tokyo. Since 2008, he has worked at National Institute of Advanced Industrial Science and Technology as a senior research scientist. His current research interest is soft matter physics including physics of surface and interface. He was a recipient of SPSJ Award for the Outstanding Paper in Polymer Journal sponsored by ZEON in 2005, CERI encouraging award for young scientist in the society of rubber science and technology Japan in 2012, Bridgestone soft material Frontier Award in the society of rubber science and technology Japan in 2014 and SPSJ Hitachi Chemical Award in the Society of Polymer Science, Japan in 2014. 